Pol J Med Phys Eng 2012;18(1):1-6.

PL ISSN 1425-4689

doi: 10.2478/v10013-012-0001-3

website: http://versita.com/pjmpe

Jakub Ośko ${ }^{1}$, Natalia Golnik ${ }^{1}$, Tomasz Pliszczyński ${ }^{1}$, Renata Sosnowiec $^{1}$, Marianna Umaniec ${ }^{1}$, Mieczysław Zielczyński ${ }^{1}$

\title{
Measurements of high level of iodine activity in thyroid with different radiation meters
}

\author{
${ }^{1}$ National Centre for Nuclear Research, Otwock-Świerk, Sołtana 7, Poland \\ e-mail: kuba.osko@cyf.gov.pl
}

\begin{abstract}
Iodine activity in thyroid of female patient was measured with different radiation meters in order to estimate a possibility to use them in case of radiation accident. Two series of measurements were performed - first after diagnostic and second after therapeutic administration of iodine to the patient. The isotope activities were higher than those registered during routine radiation monitoring and similar to the activities which could be registered after radiation accident. The studies showed that simple dose rate meters may serve for identification and selection of contaminated persons which should be later subjected to the measurements with especially dedicated equipment. The initial measurements can be performed outside laboratory.
\end{abstract}

Key words: radioiodine, nuclear medicine, radiation protection

\section{Introduction}

Iodine I-131 is one of radioisotopes, which are most commonly used in medicine, both for diagnostics and therapy. As a consequence, there is a large group of people who are occupationally exposed to internal uptake of iodine. This group includes medical staff of nuclear medicine departments and employees dealing with radioactive iodine production, transport and waste management. All of them need to be monitored for internal contamination with iodine by measuring the iodine activity gathered in thyroid. In several cases, mostly for diagnostics, there is also a need to measure radioactive iodine activity in a patient's thyroid. Usually, a scintillation detector is used for such measurements. The patient (or an employee) is sitting on a chair or lying on a couch and the detector is placed in front of the neck at an adequate distance, L. The detection efficiency, $\varepsilon$, has to be predetermined by calibration of the counter placed for the measurements at the certain distance $\mathrm{L}$ and detector "is looking" directly to the neck. Such arrangement is called here "geometry L cm.

Most measurements for radiation protection are performed at low activities gathered in thyroid, which do not exceed few hundreds of kBq. Therefore, the typical 
radiation protection procedures are designed for such level of the isotope activity. The distance between detector and human neck is usually set to $10-20 \mathrm{~cm}$. Activity gathered in thyroid after diagnostic administration is somewhat higher than during routine occupational exposure.

The situation is different in case of the measurements after therapeutic administration of iodine or after severe radiation accident, when the iodine activities can be much higher. Typical measurement geometry used for radiation monitoring becomes unsuitable because very high count rate at short distance between detector and human neck can cause too large uncertainties. The easiest way to improve the measuring condition is to increase the distance between detector and neck, however, the method of measurements in such unusual geometry should be developed.

In this work, the iodine activity in thyroid of female patient was measured with different radiation meters and the results were compared with the iodine retention curve.

\section{Materials and Methods}

The case analysis in this paper is performed for a female patient subjected to thyroid diagnostics and therapy. During diagnostic treatment, the patient was administered orally with $50 \mu \mathrm{Ci}(1,85 \mathrm{MBq})$. According to ICRP model, the expected effective dose for adult person was in this case $40,7 \mathrm{mSv}$ [1]. The iodine activity orally administered during therapeutic treatment was $555 \mathrm{MBq}$. The absorbed doses were in both cases much higher than doses which are usually recorded for occupationally exposed persons during routine work [2].

Two measuring series were provided for the studied patient - the first one at 4th, 5th and 8th day after the diagnostic administration of iodine and the second at 26th, 33rd, 47th, 80th and 87th day after the therapeutic treatment.

The measurements were performed with spectrometric equipment and with dose equivalent rate meters. A thyroid counter (marked here as LPT), which is used in NCBJ for routine radiation monitoring, served here as a main instrument. It could be considered also as a reference one, if used in proper geometry and within the reasonable counting rate range. The counter is equipped with a $\mathrm{NaI}$ scintillation detector mounted in the SS-33W52 counter (manufactured by POLON, Bydgoszcz, Poland) and connected to Tukan8k multichannel analyzer. The second spectrometric instrument was InSpector 1000 - a mobile spectrometer with NaI scintillation detector, manufactured by Cannberra-Packard. This instrument was also used as a dose rate meter. Other dose equivalent rate meters were: ECO C and FH40, both manufactured by POLON, Poland. Generally, no one of them is suitable for determination of radionuclide activity in human body. In this work, they were used with the aim to check whether they could be used for fast and simple estimation of the internal contamination evel in emergency situations, without determination of the effective dose. 
The measurements with dose equivalent meters were performed with the detectors directly at the neck $(0 \mathrm{~cm}$ geometry) and at the distance of $60 \mathrm{~cm}$. Spectrometric measurement were performed at the distance of $60 \mathrm{~cm}$. The measurements with Thyroid Counter were performed also in standard LPD geometry $12 \mathrm{~cm}$. This geometry is used in routine monitoring for measurements at lower level of activity. Earlier studies showed that dead time and associated uncertainties are too high for accurate measurement at higher activities [3].

Natural background was subtracted from the registered spectra.

\section{Results and discussion}

The results of dose rate measurements after diagnostic administration of iodine are shown in Table 1 and in Figure 1. The measurements at 4th day were performed at activity which was too high some measurements, so the readings were out of range for these instruments. Dose equivalent meter ECO C, which contains a large Geiger-Muller counter, indicated the highest values, while FH40, the instrument with small proportional counter, showed the lowest values in both measuring positions.

Table 1. The dose rates from iodine in thyroid after diagnostic administration, measured in two distances from the neck $[\mu \mathrm{Sv} / \mathrm{h}]$

\begin{tabular}{lccc}
\hline Instrument & \multicolumn{3}{c}{ Day after administration of iodine } \\
& 4 & 5 & 8 \\
\hline & & Geometry $60 \mathrm{~cm}$ & $0,15 \pm 0,02$ \\
InSpector & $0,36 \pm 0,04$ & $0,31 \pm 0,03$ & $0,14 \pm 0,01$ \\
FH40 & $0,29 \pm 0,01$ & $0,26 \pm 0,01$ & $0,34 \pm 0,05$ \\
ECO C & out of range & $0,47 \pm 0,07$ & $15,09 \pm 1,5$ \\
& & Geometry $0 \mathrm{~cm}$ & $11,39 \pm 0,06$ \\
\cline { 2 - 4 } InSpector & out of range & $26,93 \pm 2,7$ & $17,43 \pm 0,09$ \\
FH40 & out of range & & \\
\cline { 2 - 4 }
\end{tabular}

InSpector 1000 registered also the energy spectrum which made it possible to determine the activity of iodine. For this paper the number of counts in $364 \mathrm{keV}$ peak area was used as the measurement result, however, at present the values are in arbitrary units, because there was no efficiency calibration done before the measurements. Therefore, not the absolute values but their change in time is of interest in this work. In order to present all the results in the same way, they were normalized and the result at 5 th days was assumed to be 1 (see Figure 1). For comparison, also the theoretical retention curve after ingestion [6] and the results of measurements with Thyroid Counter in standard geometry, with (LPT(d)) and without (LPT) regarding of thyroid depth in the neck [3], can be seen in the same Figure. 


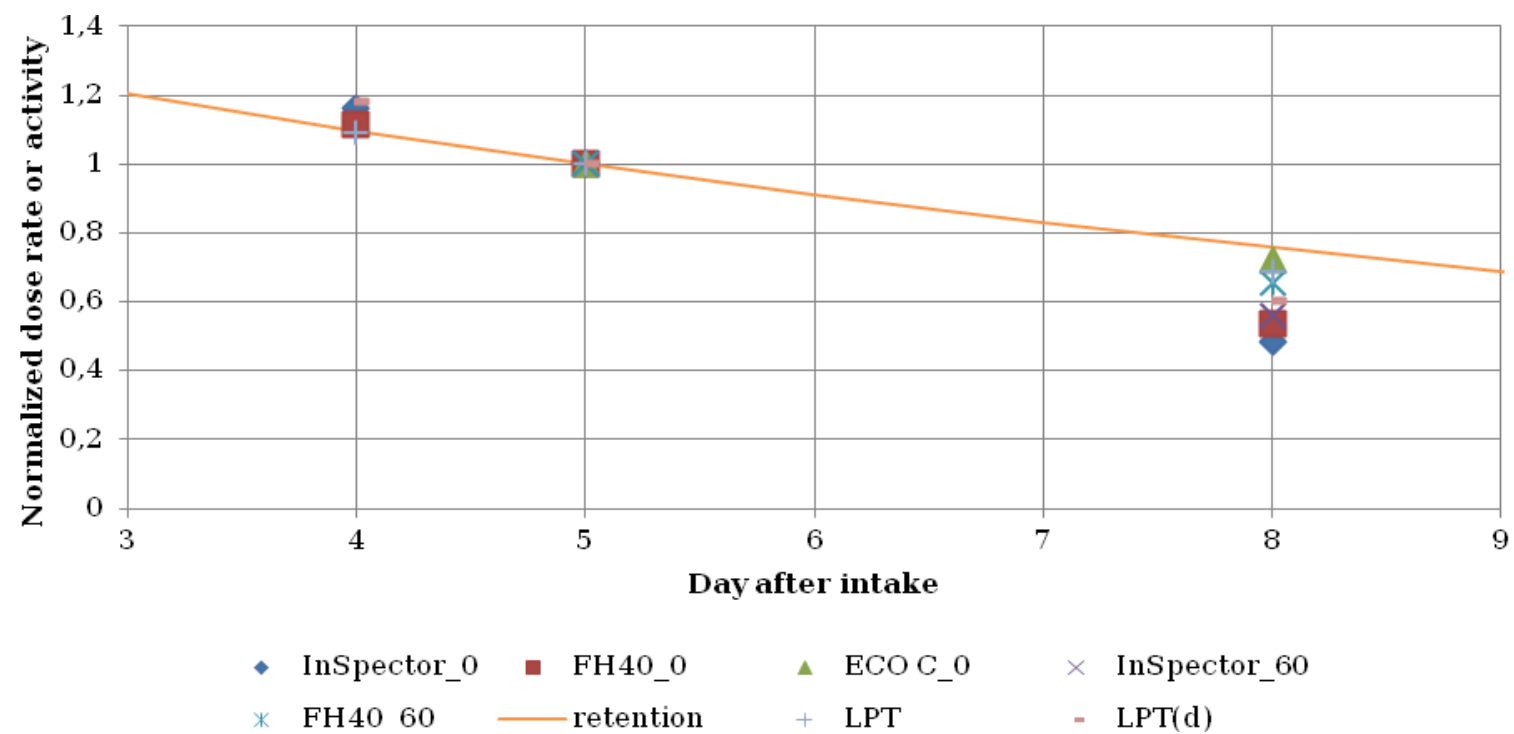

Figure 1. Normalized readings of different instruments, recorded in the measurements after diagnostic administration of iodine. Detectors are described as "Detector'sName_Geometry".

It can be seen from the Figure 1, that all the instruments showed faster removal of iodine, than expected from the retention curve. For LPT, the difference is small and can be easy attributed to physiological spread in the iodine retention in human bodies. Much larger differences in readings of other detectors appeared most likely because the instruments were purposely used in "emergency" geometry without collimators, so they measured a signal from not well defined part of the body. It can be seen that the worst result differs from the reference one by less than 50\%. Surprisingly, the dose rate results with InSpector 1000 are the most incorrect in both geometries, compared to theoretical retention function, but even these results can be considered as acceptable in internal dosimetry.

The results of the second series of measurements are presented in Table 2 and Figure 2. As previously, they are also normalized and the results at the day 33 after the iodine administration were assumed to be 1. The results presented in Figure 2 are for $60 \mathrm{~cm}$ geometry and $12 \mathrm{~cm}$ geometry for the thyroid counter LPT.

It is clearly seen from the Table 2 that in this case, the measurements in geometry $0 \mathrm{~cm}$ gave inconsistent results, because of too high dose rate. On the other hand, the measurements on the days 80 and 87 were performed at low dose rate and again somewhat larger differences between the readings are observed. 
Table 2. The dose rate from iodine in thyroid after therapeutic administration, measured in two distances from the neck $[\mu \mathrm{Sv} / \mathrm{h}]$

\begin{tabular}{lccccc}
\hline \multirow{2}{*}{ Instrument } & \multicolumn{5}{c}{ Day after administration of iodine } \\
& 26 & 33 & 47 & 80 \\
\hline \\
\cline { 2 - 5 } InSpector & $2,5 \pm 0,3$ & $1,2 \pm 0,1$ & Geometry $60 \mathrm{~cm}$ & \\
FH40 & $3 \pm 0,2$ & $1,3 \pm 0,05$ & $0,27 \pm 0,03$ & $0,04 \pm 0,005$ & $0,06 \pm 0,005$ \\
ECO C & $3,8 \pm 0,6$ & $1,4 \pm 0,2$ & $0,40 \pm 0,06$ & $0,14 \pm 0,02$ & $0,016 \pm 0,02$ \\
\cline { 2 - 6 } & \multicolumn{5}{c}{ Geometry 0 cm } \\
InSpector & $1300 \pm 130$ & $220 \pm 22$ & $16,5 \pm 1,7$ & $0,52 \pm 0,05$ & $0,22 \pm 0,02$ \\
FH40 & $180 \pm 9$ & $90 \pm 5$ & $21,3 \pm 1,1$ & $0,45 \pm 0,07$ & $0,16 \pm 0,02$ \\
ECO C & $75,8 \pm 11$ & $91,9 \pm 14$ & & & \\
\hline
\end{tabular}

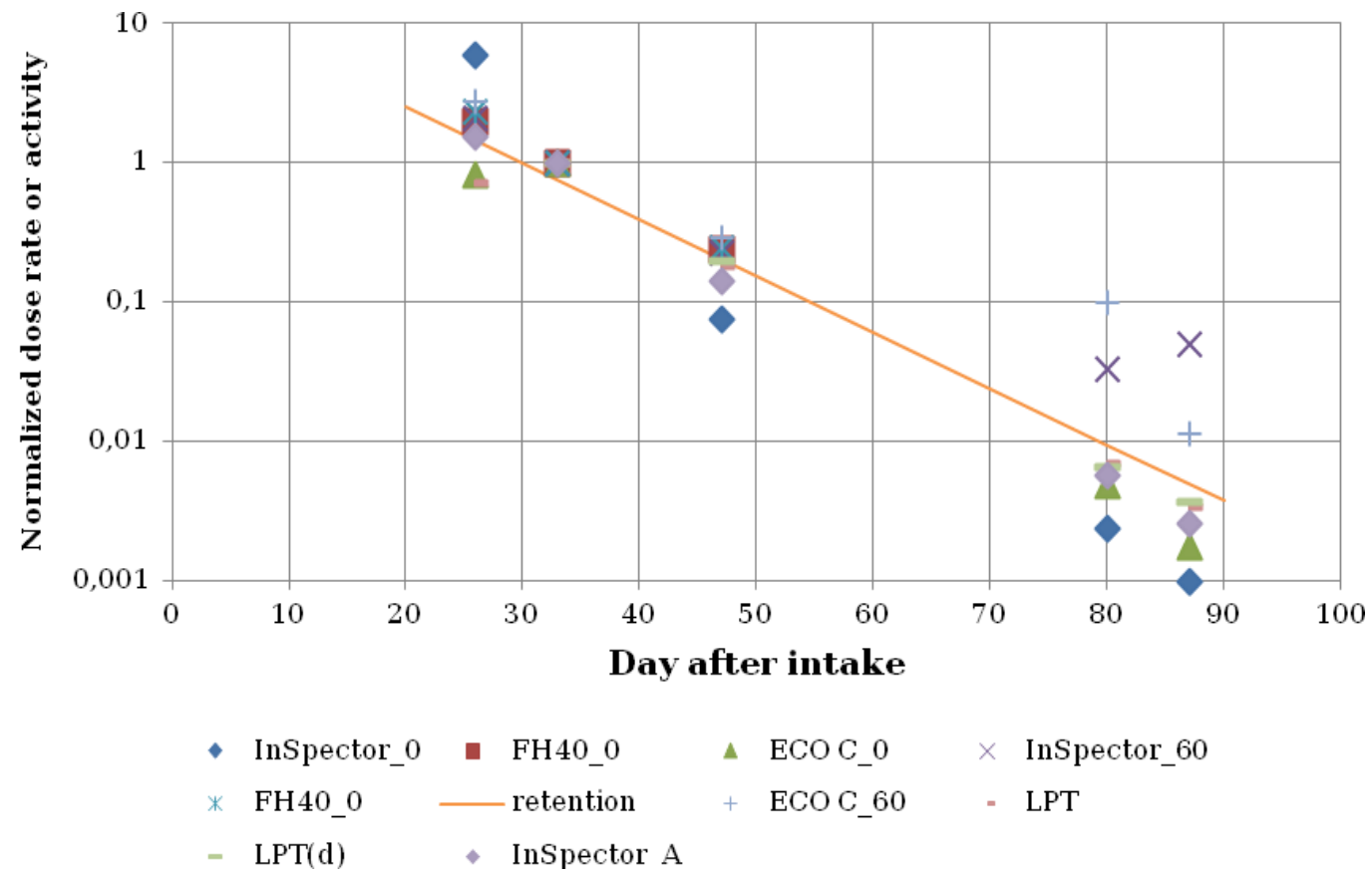

Figure 2. Normalized readings of different instruments, recorded in the measurements after diagnostic administration of iodine. Detectors are described as "Detector'sName_Geometry".

\section{Conclusions}

The results showed that most of the measuring devices used in this work can be used for initial estimation of internal contamination with iodine I-131. These measurements can be performed out of laboratory without additional shielding.

Registered results are close to theoretical retention function within large range of activity. This confirms that simple measuring devices can be used in case of radiation accident to select contaminated people for accurate measurements in laboratory. This 
would make it possible to limit the number of persons who should pass through special check in laboratory conditions.

The measuring devices used here, were calibrated for this type of measurements. Necessary calibration will be done during further studies.

There is also a need to remember that in case of thyroid measurements if the distance between neck and detector is long, the measurements results will appear as a sum of contributions from all radioisotopes gathered in human body.

\section{Acknowledgements}

The work was supported by The National Centre for Research and Development under project SP/J/6/143339/11.

\section{References}

[1] Council of Ministers of 18 January 2005 on the radiation dose limits (Dz. U. nr 20, poz. 168)

[2] J. Ośko, N. Golnik, K. Ciszewska, Internal dosimetry at the Institute of Atomic Energy POLATOM in Poland, Third European IRPA Congress, June 14-18, 2011 Helsinki, Finland - Proceedings, p. 1436-1441 (2011)

[3] J. Ośko, Spectrometric measurements of the radioactive isotopes uptake in thyroid, PhD's thesis, Warsaw Technical University, Faculty of Mechatronics, 2008, Polish

[4] L. Królicki, Nuclear Medicine, Fundacja im. L. Rydygiera, 1996, in Polish

[5] Ośko J., Golnik N., Pliszczyński T. Spectrometric measurements of iodine and technetium activity in thyroid, NUCL INSTRUM METH A, 580 (578-581), 2007

[6] ISO FDIS 27048:2010 Radiation protection - Dose assessment for the monitoring of workers for internal radiation exposure 